\title{
Rats Development of Contactless Thermal Detector for Animal: Comparison of Three Sensor Types
}

\author{
Ridi Arif ${ }^{1 *}$, Koekoeh Santoso ${ }^{2}$, Dhani S. Wibawa ${ }^{3}$ \\ ${ }^{I}$ Department of Animal Infectious Diseases and Veterinary Public Health, Faculty of Veterinary Medicine, IPB \\ University \\ ${ }^{2}$ Department of Anatomy, Physiology, and Pharmacology, Faculty of Veterinary Medicine, IPB University \\ ${ }^{3}$ Surfactant and Bioenergy Research Center, IPB University \\ *Corresponding author.Email: ridiarif@gmail.com
}

\begin{abstract}
One of the important physiological parameters is body temperature, and the increased level is an early indicator of infection. Therefore, it is necessary to develop temperature detection in animals using contactless methods and with precise results. This study aimed to compare 3 types of IR sensors for the body temperature check without contact in animals. The three sensors are the Thermo gun sensor type, MLX90614, and AMG8833. The MLX90614 and AMG8833 are used by ensuring a connection with the Arduino Uno microcontroller. The script and libraries used were followed the standard code provided by Arduino. Furthermore, the data from the 3 sensors were compared with the FLIR C2 professional thermal camera and the contact temperature measurement was carried out with a thermometer. The results of measurements using 3 types of sensors showed that the thermo gun sensor was not suitable for non-contact temperature check in animals since it cannot be calibrated. The MLX906144 and AMG8833 sensors can be used as non-contact thermometers in animals by optimizing and modifying the script code or adding circuit components. To obtain the temperature measurement optimization formula, it should be repeated with a larger number of trials, while paying attention to the factor of ambient change.
\end{abstract}

Keywords: Ambient change, Animal, IR sensor, Thermal detector

\section{INTRODUCTION}

One of the most important physiological parameters is body temperature which in warmblooded animals it kept within a narrow range. Furthermore, it indicates a good physiological status, normal temperature range, and the metabolic processes of the body run well because enzymes perform their functions optimally [1]. The impaired enzyme performance lead to the increased body temperature resulted in interfere the physiological functions of the body. The increase body temperature caused by several factors and the most common cause is infection [2]. Therefore, the ability to quickly and accurately observe an increase in body temperature may also detect early infection.

The Covid-19 pandemic makes body temperature checking is important. The temperature checking helps to detect the presence of fever as early signal of any kind of infection. Since Covid-
19 is transmitted through close contact, the temperature checking process needs to be conducted without any contact. For the benefit of humans, temperature screening is usually conducted using an Infra-Red thermo gun [3]. Furthermore, non-contact temperature checks on animals are still generally used for screening purposes. This is evident in wild animals [4] and livestock populations $[5,6]$. The use of thermal cameras to check the temperature in animals on a non-contact basis also supports the animal welfare [7]. On the contrary, temperature checks for animals have not been developed in more detail by the contactless method. This is necessary due to reports that several animal species may be infected with Sars Corona Virus 2 [8]. However, animals infected are not the main source of transmission in the Covid-19 pandemic case [9] since they have the possibility to transmit to both humans and animals. Therefore, it is necessary to develop a tool to check the temperature without contact with animals. 
In animals, body temperature is usually measured by inserted a thermometer into the area of the anus since it provides an accurate internal measurement. To develop a method of measuring body temperature without contact, it is necessary to conduct an experiment related to the scan location, the type of sensor used, the optimum scanning distance, and several other variables. This is a preliminary study that aims to compare several types of body temperature sensors for animals in order to obtain comparative data and recommended types of sensor tools.

\section{MATERIALS AND METHODS}

The tools used include a thermometer (contact), thermo gun, FLIR C2 thermal infrared camera, AMG8833 temperature sensor, MLX90614 temperature sensor, Arduino microcontroller, and Arduino IDE software, and the experimental animals used were three healthy sheep.
Furthermore, temperature measurements on the animals were conducted using all experimental tools. The results obtained using a contact thermometer are considered to be the most accurate and used as a reference/golden standard. Measurements are carried out on one area around the anus using a thermogun. It is conducted at the scanning distance following the recommendations of the instrument used. The measurement using the FLIR C2 thermal camera was conducted by scanning the temperature in the anal region. Furthermore, the AMG8833 and the MLX90614 sensors are made by using an Arduino microcontroller, and the script code used is the officially available default program.

\section{RESULTS AND DISCUSSION}

The results of the temperature measurement are presented in Table 1.

Table 1 Temperature measurement $\left({ }^{\circ} \mathrm{C}\right.$ ) and factorial analysis results of temperature check

\begin{tabular}{|l|l|l|c|c|}
\hline \multirow{2}{*}{ Factor } & \multicolumn{3}{c|}{ Sheep (f1) } \\
\cline { 2 - 5 } & Thermogun & $36.75 \pm 0.07$ & $38.15 \pm 0.07$ & $37.90 \pm 0.28$ \\
\cline { 2 - 5 } Types of tools \\
(f2) & FLIR & $39.45 \pm 0.21$ & $39.45 \pm 0.35$ & $40.05 \pm 0.07$ \\
\cline { 2 - 5 } & AMG8833 & $32.19 \pm 0,08$ & $31.55 \pm 0.71$ & $32.03 \pm 0.23$ \\
\cline { 2 - 5 } & MLX90614 & $36.75 \pm 0.65$ & $36.43 \pm 1.07$ & $34.16 \pm 0.18$ \\
\cline { 2 - 5 } & Therm. contact & $39.20 \pm 0.00$ & $39.25 \pm 0.07$ & $39.40 \pm 0.00$ \\
\hline \multirow{3}{*}{ p-value factor } & Sheep & 0.376 & \\
\cline { 2 - 5 } & Tools & 0.00 & \\
\cline { 2 - 5 } & Sheep*Tool & 0.00 & \\
\hline
\end{tabular}

Description: Factor 1 (f1) is the type of sheep; factor 2 (f2) is the type of tool used

In this analysis, a factorial test was conducted on the first factor using sheep as a sample, while the second was the type of instruments used. The factor consists of 3 levels, sheep A, B, and C; while the tool consists of 5 levels, namely the thermo gun, FLIR C2, AMG sensor, MLX sensor, and thermometer contact. The analysis results showed that the sheep factor used was obtained at a p-value of $0.376 \quad(\mathrm{p}>0.05)$. Therefore, the temperature measurements for the three types of sheep tested were relatively the same, and they have the same body temperature range. Conversely, the analysis of the tool factor used obtained a very significant $\mathrm{p}$-value $(\mathrm{p}<0.00)$. This shows that the tool used to measure temperature obtained a significantly different result. In addition, it was reported that the p-value in the interaction between the factor of sheep and the tools used was also very significant. Therefore, there is an interaction between the experimental sheep factor and the tool used towards the temperature measurement results obtained.

Furthermore, an analysis was conducted to compare the test result of each type of tool used, it is presented in Table 2.

Table 2 Results analysis of temperature checks

$\left({ }^{\circ} \mathrm{C}\right)$ and its error percentage

\begin{tabular}{|l|l|l|}
\hline Types of tools & Mean \pm SD & Errors (\%) \\
\hline Thermogun & $37.60 \pm 0.68^{\mathrm{b}}$ & $66.13 \%$ \\
\hline FLIR & $39.65 \pm 0.36^{\mathrm{a}}$ & $14.4 \%$ \\
\hline AMG8833 & $31.93 \pm 0.45^{\mathrm{c}}$ & $289.05 \%$ \\
\hline MLX90614 & $35.78 \pm 1.38^{\mathrm{d}}$ & $137.62 \%$ \\
\hline Therm. contact & $39.28 \pm 0.09^{\mathrm{a}}$ & - \\
\hline
\end{tabular}

Description: Different superscript represent significant differences $(\mathrm{p}<0.05)$ 
This study showed a significant variation, and the checking results were obtained by contact using a body thermometer. Temperature measurements were conducted through anal checking, and the obtained results were used as a reference to best describe internal body temperature. Meanwhile, the results of this contact thermometer checking obtained a temperature measurement value of $39.28 \pm 0.09{ }^{\circ} \mathrm{C}$. On the contrary, the checking results using the IR FLIR C2 camera showed a slightly higher value of $39.65 \pm 0.36{ }^{\circ} \mathrm{C}$ but were not significantly different. The results using the thermogun, MLX and AMG sensors were $37.60 \pm 0.68{ }^{\circ} \mathrm{C}, 35.78 \pm 1.38{ }^{\circ} \mathrm{C}$, and $31.93 \pm 0.45{ }^{\circ} \mathrm{C}$, with significant values differing from one to the other respectively. A comparison with the contact thermometer showed that the error rate from lowest to highest value is FLIR C2 IR camera (14.4\%), Thermogun (66.13\%), MLX sensor (137, $62 \%)$, and AMG sensor (289.05\%).

The best result that most closely approximates the measurement of the contact thermometer is the noncontact checking with the FLIR C2 IR camera. Also, the results using the FLIR thermal camera have the advantage of being able to conduct an in-depth scan. However, to obtain the temperature value, the thermometer should be exposed to a single point of measurement. The FLIR C2 IR camera is a professional tool for performing a wide variety of temperature scans. In the medical field, some practitioners use this tool to scan the process of wound healing [10].

Another device that obtained a good result was thermogun, however, it was significantly different from FLIR C2. This device uses an infrared sensor similar to the MLX 90614. Thermogun is commonly used to measure temperature in humans without contact [11]. This study showed that the use of thermogun in animals still has a big error. Therefore, it needs to be optimized or recalibrated prior to be used to measure animal temperature.

The subsequent results show that the MLX 90614 sensor connected to the Arduino obtained an error value of $137.62 \%$ using the default script code. This value is still better than the AMG 8833 sensor with an error value of $289.05 \%$. Both of them take a long time to show stable measurement results when used. The AMG 8833 sensor is widely used as a temperature scanner and may be combined with other modules. For example, it is used in manufacturing drones equipped with the Internet of Things (IoT) system [12]. Furthermore, it was also developed for temperature detection in the medical field because of its affordable price, and it has the largest error value in this study. In contrast, the study by [5] which also used AMG 8833 obtained good results in making a tool for the detection of pathological events through temperature scanning. A Raspberry Pi microcontroller was used, and therefore the optimized code formulation yielded precise results. Furthermore, the use of the Arduino microcontroller is less precise because the value is very far from the standard golden value.

Conversely, body temperature measured using a detecting tool is slightly affected by the humidity of the environment. This is because the heat in the body quickly disappears when the ambient temperature is low and humidity is high due to the increased rate of evaporation. During this study, it was reported that the ambient temperature was $30.7^{\circ} \mathrm{C}$ with a relative humidity of $60.7 \%$. This factor can be included in the script code preparation process as one of the variables affecting the measurement results. However, this factor was not considered a calculated variable because this experiment only used standard code available in the Arduino IDE software library.

\section{CONCLUSION}

The use of an IR FLIR C2 camera for temperature measurement in animals is usually preferred because it has a small error percentage. On the contrary, the use of a Thermogun to directly measure the temperature of animals is not recommended due to its large error. Before being used to measure the temperature of animals, it needs to be calibrated. The MLX 90614 and AMG 8833 sensors along with Arduino are a form of basic series or prototype that should be developed to obtain an optimal measurement result. To perform this optimization, experiments with more animals are needed to obtain a formula in the form of script code. Therefore, the measurement results are closer using a contact thermometer.

\section{AUTHORS' CONTRIBUTIONS}

RA, KS, and DSW are equally contributed to this work.

\section{ACKNOWLEDGMENTS}

The author is grateful to the Directorate General of Higher Education, Ministry of Research, Technology, and Higher Education for providing grants through the 2020 Higher Education Leading Research scheme no 2761/IT3.L1/PN/2020.

\section{REFERENCES}

[1] S.F. Morrison, Central control of body temperature, F1000Res. 5 (2016) 1-0, F1000 Faculty Rev-880. DOI: 10.12688/f1000research.7958.1S.F.

[2] E.A. Tansey, C.D. Johnson, Recent advances in thermoregulation. Adv. Physiol. Educ. 39(3) 
(2015) 139-148. DOI: 10.1152/advan.00126.2014.

[3] H.Y. Chen, A. Chen, C. Chen, Investigation of the Impact of infrared sensors on core body temperature monitoring by comparing measurement sites. Sensors 20 (2020) 1-17. doi: 10.3390/s20102885

[4] J. Cilulko, P. Janiszewski, M. Bogdaszewski, E. Szczygielska, Infrared thermal imaging in studies of wild animals. Eur. J. Wild Res. 59 (2013) 17 23.

[5] U.A Jayalatsumi, F. Naaz, K. Sravani, A.Anusha, A. Vasavi, A low cost thermal imaging system for medical diagnostic applications. IJET 7(3.27) (2018) 314-317.

[6] R. Jeelani, Thermal imagery for monitoring livestocks. IJLSAS. 1(1) (2019): 58-69.

[7] I.A. Naas, R.G. Garcia, F.R. Caldara, Infrared thermal image for assessing animal health and welfare. J. Anim. Behav. Biometeorol. 2(3) (2014) 66-72.

[8] T. Opriessnig, Y.W Huang, Update on possible animal sources for COVID-19 in humans. Xenotransplantation 27(3) (2020) 1-4.

[9] N.M.A. Parry, COVID-19 and Pets: When pandemic meets panic. FSI Reports 2 (2020) 100090.

[10] S. Songsak, K. Kiranantawat, K. Sananpanich, Detection of perforators using smartphone thermal imaging, Plast. Reconstr. Surg. Glob. Open 4(5) (2016) E722. DOI: 10.1097/GOX.0000000000000715

[11] S. Shazia, N. Akhter, R. Manza, Current trends in the application of thermal imaging in medical condition analysis. IJITEE 8(8) (2019) 27082712 .

[12] K. Sruthi, G. Anil, Agricultural drone using IoT. IJTSRD 2(4) (2018) 2006-2009. 